\title{
Totally Blind Channel Estimation for OFDM on Fast Varying Mobile Radio Channels
}

\author{
Marc C. Necker and Gordon L. Stüber
}

\begin{abstract}
A new blind channel estimation scheme for OFDMsystems is proposed based on the ML-principle. By avoiding the use of second- and higher-order statistics, a very fast convergence rate is achieved. A novel approach is also proposed for resolving the phase ambiguity of the blind channel estimate without the need for any reference symbols. The approach combines different modulation schemes on adjacent subcarriers, such as 3-PSK and QPSK, to resolve phase ambiguity. Simulations were performed for mobile radio environments with high Doppler frequencies and short-to-medium delay spreads. The achieved performance is comparable to that of pilot-based channel estimation for the case of QPSK-modulation.
\end{abstract}

Index Terms-OFDM, blind channel estimation, QPSK, 3PSK, 5-PSK

\section{INTRODUCTION}

Orthogonal Frequency Division Multiplexing (OFDM) is of great interest for digital communication on mobile multi-path fading channels. To perform coherent demodulation it is necessary to have knowledge of the time-variant channel transfer function. In an OFDM-system, the channel transfer function can conveniently be estimated using a two-dimensional grid of pilot symbols [1]. The Digital Video Broadcasting Terrestrial (DVB-T) standard [2] is one such example. However, channel capacity is wasted due to the transmission of the pilot symbols in these systems. An alternative is to use differential phaseshift keying (DPSK) and differentially coherent demodulation. This has been implemented successfully in the Digital Audio Broadcasting standard [3]. However, differential detection leads to an $E_{b} / N_{o}$ loss of about $2 \mathrm{~dB}$ for an AWGN channel and a larger loss for fading channels [4]. Hence, it is desirable to use coherent demodulation while being able to determine the channel transfer function without the need for pilot symbols, a technique known as blind channel estimation. Much research has focused on blind channel estimation, but the performance has not been comparable to that of pilot-based channel estimation.

Most existing blind channel estimation methods are based on second or higher order statistics. Examples of statistical blind channel estimation techniques include those using correlation methods [5] and cumulant fitting schemes [6] and [7]. In [8], the asymptotic performance and fundamental limitations of blind estimators based on second order statistics has been

M. C. Necker is with the Institute of Communication Networks and Computer Engineering, University of Stuttgart, Pfaffenwaldring 47, 70569 Stuttgart, Germany. Ph. +49 711685 7963, Fax. +49 7116857983 (EMail: necker@ikr.uni-stuttgart.de). Work was done while he was with the School of Electrical and Computer Engineering, Georgia Institute of Technology.

G. L. Stüber is with the School of Elextrical and Computer Engineering of the Georgia Insitute of Technology, Atlanta, GA 30332-0250. Ph. +1 404 894 2923, Fax. +1 4048947883 (Email: stuber@ece.gatech.edu). investigated. While methods based on higher order statistics can recover both the magnitude and phase of the channel transfer function, those based on second order statistics can only recover the magnitude. Afterwards, the phase can be recovered if and only if the received signal is cyclostationarity. Other blind channel estimation methods developed for OFDM take advantage of the redundancy introduced by the cyclic prefix, e.g., [9], [10]. In [11], blind channel estimation was investigated for IEEE 802.11a. In their paper, the authors used a finite alphabet approach and clustering of subcarriers. In any case, the phase information is recovered but there is still a phase ambiguity. To recover the phase information completely, additional reference symbols can be inserted into the data stream. Although only a few reference symbols are needed, the charm of blind channel estimation is lost.

Statistical blind channel estimation approaches have a slow convergence rate, making them unsuitable for mobile radio channels. Moreover, they only work with continuous transmission and fail for burst transmission. In contrast to the statistical methods, Chotikakamthorn and Suzuki applied a deterministic approach based on the maximum likelihood (ML)-principle to OFDM systems [12]. This method has the advantage of producing a channel estimate from a single received OFDM symbol. Thus, it performs well for mobile radio channels, and is suitable for continuous and burst traffic alike. Its principal drawback is the huge computational complexity needed to execute the maximization operation embedded in the algorithm. Also, the channel estimate still has a phase ambiguity.

In this paper we modify the basic ML-method from [12] for the case of PSK signals. It is shown for the noise-free case that blind channel estimation can be achieved by considering only two data symbols which are adjacent within one received OFDM data symbol if the delay spread of the channel impulse response stays within certain limits. This concept is further extended to the noisy case. A suboptimal approach for performing the maximization operation of the ML-method is presented. A variation of the algorithm is developed that yields a low-complexity blind channel estimator which can estimate the channel from a single OFDM symbol. It is shown that the estimator can be improved by using iterations and exploiting the time-domain correlation of the channel transfer function.

We also present a novel approach for resolving the phase ambiguity of the channel estimate. By combining two different modulation schemes on adjacent OFDM subcarriers, a unique channel estimate can be obtained at the receiver. In particular, we investigate the combination of QPSK and 3-PSK resp. 5-PSK. Our approach completely recovers the complex channel gain (amplitude and phase), without requiring any reference symbols at all. Thus the proposed channel estimator 
performs a true blind channel estimation.

The remainder of the paper is as follows. Section II develops our maximum likelihood blind channel estimator from first principles. In section IV we introduce the concept of combined PSK modulation schemes to resolve the phase ambiguity of the channel estimate. Section III presents the suboptimal approach for solving the maximization operation. Finally, Section V presents applications and simulation results for the proposed blind channel estimation method.

\section{The Maximum Likelihood Blind Channel ESTIMATOR}

Consider an OFDM modulator with $N$ subcarriers and qPSK-modulated data symbols. During an arbitrary OFDM symbol period, the transmitted data symbol vector is

$$
\tilde{\mathbf{b}}=\left[\begin{array}{llll}
b_{0} & b_{1} & \cdots & b_{N-1}
\end{array}\right],\left|b_{n}\right|=1 .
$$

The OFDM modulator computes the inverse discrete Fouriertransform (IDFT) of the frequency domain data vector $\tilde{\mathbf{b}}$ to yield the time domain vector

$$
\mathbf{W}=\left[\begin{array}{llll}
W_{0} & W_{1} & \cdots & W_{N-1}
\end{array}\right]
$$

where

$$
W_{k}=\sum_{v=0}^{N-1} b_{v} e^{\frac{j 2 \pi v k}{N}} .
$$

Ignoring the cyclic guard interval, the samples $W_{k}$ are then passed through a D/A converter to generate the transmitted complex envelope $\tilde{s}(t)$.

Let $h(t, \tau)$ denote the time-variant channel impulse response. Disregarding any non-linearities associated with RF processing, the received complex envelope is

$$
r(t)=\int_{-\infty}^{\infty} h(t, y) \tilde{s}(t-y) d y+n(t) .
$$

The waveform $r(t)$ is sampled at epochs $t=k T_{s}$ to yield the received vector

$$
\mathbf{r}=\left[\begin{array}{llll}
r_{0} & r_{1} & \cdots & r_{N-1}
\end{array}\right],
$$

where $T_{s}$ is the duration of one data symbol $b_{n}$. Assuming that $h(t, \tau)$ remains approximately constant for the OFDM symbol duration, i.e., there is no inter-carrier interference (ICI), then the discrete Fourier-transform (DFT) of $\mathbf{r}$ is

$$
z_{k}=H_{k} \cdot b_{k}+N_{k},
$$

where $N_{k}$ is AWGN and $H_{k}$ is the sampled channel transfer function

$$
H_{k}=H(t, k \Delta \omega), \quad H(t, \omega)=\mathcal{F}_{\tau}\{h(t, \tau)\},
$$

where $\Delta \omega=2 \pi / N T_{s}$.

Let $\mathbf{b}=\left[\begin{array}{llll}b_{0} & b_{1} & \cdots & b_{M-1}\end{array}\right]$ be a vector with a selection of $M$ data symbols on subcarriers with a regular spacing of $\kappa \Delta \omega$ radians, with $\kappa$ being positive integer. The received frequency domain signal vector $\mathbf{z}=\left[\begin{array}{llll}z_{0} & z_{1} & \cdots & z_{M-1}\end{array}\right]$ on these subcarriers can be conveniently written in matrix notation. To do so, let $\mathbf{A}_{d}$ be the DFT matrix, where

$$
\begin{aligned}
\mathbf{A}_{d} & =\left[\begin{array}{llll}
\mathbf{a}_{d, 0} & \mathbf{a}_{d, 1} & \cdots & \mathbf{a}_{d, L-1}
\end{array}\right], \\
\mathbf{a}_{d, m} & =\left[\begin{array}{llll}
1 & e^{-j m \Delta \omega T_{s}} & \cdots & e^{-j m \kappa \Delta \omega T_{s}(M-1)}
\end{array}\right]^{T} .
\end{aligned}
$$

The received signal vector $\mathbf{z}$ can be written as

$$
\mathbf{z}=\mathbf{B} \mathbf{A}_{d} \mathbf{h}+\mathbf{N}=\mathbf{B H}+\mathbf{N},
$$

where $\mathbf{h}$ is a length- $L$ vector of taps for the discrete-time channel impulse response, $\mathbf{H}=\left[\begin{array}{llll}H_{0} & H_{1} & \cdots & H_{M-1}\end{array}\right]$ is the vector of the channel transfer function coefficients, $\mathbf{N}=$ $\left[\begin{array}{llll}N_{0} & N_{1} & \cdots & N_{M-1}\end{array}\right]$ is AWGN vector, and

$$
\mathbf{B}=\left(\begin{array}{cccc}
b_{0} & & & \\
& b_{1} & & \\
& & \ddots & \\
& & & b_{M-1}
\end{array}\right) \text {. }
$$

Chotikoakamthorn and Suzuki [12] show that the channel can be estimated from a single received OFDM symbol. Theorem 1 below provides the underlying basis that makes it possible to apply the maximum-likelihood principle to only one OFDM symbol.

Theorem 1 (from [12]): The channel parameters $\mathbf{h}$ and the transmitted symbols $\mathbf{b}$ are uniquely identifiable up to a scaling factor, if

$$
M>Q(L-1),
$$

with $Q$ being the number of $b_{i} / b_{j}$ with distinct values for all possible permutations of symbols $b_{i}$ and $b_{j}$ of the symbol alphabet $S$.

Theorem 1 implies that there is only one vector $\mathbf{b}$ and one vector $\mathbf{h}$ that can yield the received vector $\mathbf{r}$ in the noisefree case. If noise is present, a maximum likelihood estimator for both $\mathbf{b}$ and $\mathbf{h}$ can be constructed. If the noise $\mathbf{N}$ is white and Gaussian, the maximum likelihood estimates of $\mathbf{b}$ and $\mathbf{h}$ are those vectors that minimize the quadratic error from the received sequence $\mathbf{z}$ :

$$
\hat{\theta}=\min _{\theta}\left\|\mathbf{z}-\mathbf{B} \mathbf{A}_{d} \mathbf{h}\right\|^{2} \quad, \quad \theta:=\left[\mathbf{h}^{T}, \mathbf{b}^{T}\right]^{T} .
$$

Defining the diagonal matrix $\mathbf{Z}$ with

$$
\mathbf{Z}=\left(\begin{array}{cccc}
z_{0} & & & \\
& z_{1} & & \\
& & \ddots & \\
& & & z_{M-1}
\end{array}\right)
$$

and by exploiting the constant modulus property of PSK signals, (12) reduces to (see [12]):

$$
\begin{aligned}
\hat{\mathbf{b}} & =\max _{\mathbf{b}} \operatorname{Tr}\left(\mathbf{Z}^{*} \mathbf{A}_{d} \mathbf{A}_{d}^{H} \mathbf{Z} \mathbf{b}^{*} \mathbf{b}^{T}\right) \\
& =\max _{\mathbf{b}} \mathbf{b}^{T} \mathbf{Z}^{*} \mathbf{A}_{d} \mathbf{A}_{d}^{H} \mathbf{Z} \mathbf{b}^{*}
\end{aligned}
$$

with ${ }^{*}$ denoting the complex conjugate. Once the data symbols $b_{n}$ have been estimated by solving (14), a simple estimate of the channel transfer function can be obtained by solving

$$
\hat{\mathbf{H}}=\hat{\mathbf{B}}^{*} \mathbf{z} \text {. }
$$

In the case of q-PSK, this estimate still contains a phase ambiguity, since there are $q$ different solutions to (14) that yield the same maximum value. Also note that the calculations involve the received symbols of only one OFDM-symbol.

The optimization in equation (14) is a seemingly difficult task with no obvious solution. A brute force algorithm must 
exhaust all $2^{(M-1) \log _{2} q}$ possibilities for $\mathbf{b}$ (where $b_{0}$ can be chosen arbitrarily because of the phase ambiguity). In [12], a branch-and-bound integer programming strategy ${ }^{1}$ is applied for the case of BPSK signals. However, the algorithm still has high computational complexity, especially for long channel impulse responses and larger values of $M$. In the remainder of this section, two new theorems for the noise-free case are introduced that allow us to greatly reduce the computational complexity in solving (14) by reducing the block size $M$.

Theorem 2: By using knowledge of only the received vector $\mathbf{z}$, the channel parameters $\mathbf{H}=\left[\begin{array}{ll}H_{0} & H_{1}\end{array}\right]$ and the transmitted symbols $\mathbf{b}=\left[\begin{array}{ll}b_{0} & b_{1}\end{array}\right]$ are uniquely identifiable up to a complex scaling factor if $\left(b_{0}, H_{0}\right)$ and $\left(b_{1}, H_{1}\right)$ belong to adjacent subcarriers and $|\varepsilon|=\left|H_{1}-H_{0}\right|$ is less than half the minimum Euclidean distance between any two received signal points $z_{i}$ and $z_{j}$ in the complex plane, where $\varepsilon=H_{1}-H_{0} . A n$ equivalent requirement is that $\left|\varepsilon / H_{1}\right|<d_{\min } / 2$, where $d_{\min }$ is the minimum Euclidean distance between any two signal constellation points $b_{i}$ and $b_{j}$.

Proof: It is sufficient to show that (12) has a unique solution:

$$
\begin{aligned}
\hat{\theta} & =\min _{\theta}\left\|\mathbf{z}-\mathbf{B} \mathbf{A}_{d} \mathbf{h}\right\|^{2} \quad, \quad \theta:=\left[\mathbf{h}^{T}, \mathbf{b}^{T}\right]^{T} \\
\hat{\psi} & =\min _{\psi}\|\mathbf{z}-\mathbf{B H}\|^{2}, \quad \psi:=\left[\mathbf{H}^{T}, \mathbf{b}^{T}\right]^{T} \\
& =\min _{\psi}\left\|\left[z_{0}-H_{0} b_{0}, z_{1}-H_{1} b_{1}\right]\right\|^{2} \\
& =\min _{\psi}\left\|\left[z_{0}-H_{0} b_{0}, z_{1}-\left(H_{0}+\varepsilon\right) b_{1}\right]\right\|^{2} \\
& =\min _{\psi}\left\|z_{0}-H_{0} b_{0}\right\|^{2}+\left\|z_{1}-\left(H_{0}+\varepsilon\right) b_{1}\right\|^{2} .
\end{aligned}
$$

Due to the phase-blindness, $b_{0}$ can be chosen arbitrarily. The coefficient $H_{0}$ can then be calculated as $H_{0}=z_{0} b_{0}^{*}$. Without loss of generality, $b_{0}$ is set to 1 , yielding with (12):

$$
\begin{aligned}
\hat{b}_{1} & =\min _{b_{1}} \underbrace{\left\|z_{0}-z_{0} b_{0}^{*} b_{0}\right\|^{2}}_{\text {constant }}+\left\|z_{1}-\left(z_{0} b_{0}^{*}+\varepsilon\right) b_{1}\right\|^{2} \\
& =\min _{b_{1}}\left\|z_{1}-\left(z_{0}+\varepsilon\right) b_{1}\right\|^{2} .
\end{aligned}
$$

Since $\varepsilon$ is not known, it must be dropped from this equation. The introduced error will not affect the result if $|\varepsilon|$ is smaller than an upper bound $\varepsilon_{\text {max }}$, as illustrated in Fig. 1. If $|\varepsilon|$ is smaller than half the Euclidean distance between any two plausible noiseless received symbol points, then the solution of (17) for $\hat{b}_{1}$ remains unchanged. In other words, $\left|\varepsilon / H_{1}\right|<$ $d_{\min } / 2$. Under this condition, $\mathbf{b}$ is uniquely determined up to a complex scaling factor, and thus $\mathbf{H}$ is also known up to a complex scaling factor, c.f. (15). Theorem 2 is thereby proved

Theorem 2 basically states that two channel coefficients belonging to adjacent subcarriers can be estimated if the channel transfer function does not vary too fast in frequency. It is well known that the time-variant channel transfer function $H(t, \omega)$ is related to the time-variant impulse response by a Fourier Transform [14]. On the other hand, the time-variant impulse response $h(t, \tau)$ is directly related to the power delay profile

\footnotetext{
${ }^{1}$ The branch-and-bound technique breaks a problem into subproblems until each subproblem is easy to solve. The solution space is divided in such a way that a large number of non-optimal solutions can be rejected without the need for investigating them. See [13] for more details.
}

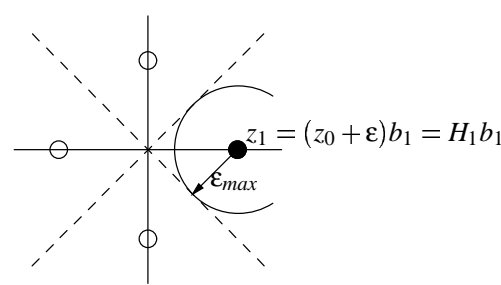

Fig. 1. Derivation of upper bound $\varepsilon_{\max }$ with QPSK as an example.

such that the shape of $|h(t, \tau)|$ plotted over time matches the shape of the power delay profile [15]. In particular, the longer the power delay profile, the faster are the variations of $H(t, \omega)$ in frequency. The restrictions imposed on $\mathbf{H}$ in Theorem 2, therefore, directly translate to conditions on the channel power delay profile. Channels having a short delay spread, such as in rural areas, are likely to fulfill the necessary condition. Likewise, channels in hilly areas with a long delay spread most likely will not meet the conditions imposed by Theorem 2 . Appendix A shows how to estimate the maximum feasible delay spread for the signaling schemes under consideration.

Just like Theorem 1, Theorem 2 holds only in the noise-free case. A blind channel estimator based on Theorem 2 is more likely to be foiled by noise than one based on Theorem 1, since fewer subcarriers are involved. The noise sensitivity can be improved by using more than two symbols leading to Theorem 3.

Theorem 3: By knowing only the received vector $\mathbf{z}$, the channel parameters $\mathbf{H}=\left[H_{0} \cdots H_{M-1}\right]$ and the transmitted symbols $\mathbf{b}=\left[\begin{array}{lll}b_{0} & \cdots & b_{M-1}\end{array}\right]$ are uniquely identifiable up to a complex scaling factor for any $M \geq 2$, if $\left(b_{0}, H_{0}\right) \cdots\left(b_{M-1}, H_{M-1}\right)$ belong to consecutive subcarriers and the channel transfer function coefficients $\mathbf{H}$ change slowly in the frequency domain, i.e. $\left|\frac{\varepsilon}{H_{n}}\right|=\left|\frac{H_{n}-H_{n-1}}{H_{n}}\right|<d_{\min } / 2, n=0, \ldots N-1$.

Proof: Theorem 3 can be proved by using Theorem 2 and induction.

Assumption Step: The channel is assumed to be uniquely identifiable for $M=2$ according to Theorem 2 .

Induction Step: Let $\mathbf{H}=\left[\begin{array}{llll}H_{0} & H_{1} & \cdots & H_{M-1}\end{array}\right]$ and $\mathbf{b}=$ $\left[\begin{array}{llll}b_{0} & b_{1} & \cdots & b_{M-1}\end{array}\right]$ be the vectors fulfilling equation (12). It is sufficient to show that by adding the elements $H_{M}$ and $b_{M}$ to both of these vectors the uniqueness of the solution is still maintained. If $H_{M}=H_{M-1}+\varepsilon$, derivations similar to those of (16) yield from equation (12):

$$
\begin{aligned}
\hat{\psi}= & \min _{\psi}\|\mathbf{z}-\mathbf{B H}\|^{2} \\
= & \min _{\psi}\left\|\left[z_{0}-H_{0} b_{0}, z_{1}-H_{1} b_{1} \cdots z_{M}-H_{M} b_{M}\right]\right\|^{2} \\
= & \min _{\psi} \underbrace{\left\|z_{0}-H_{0} b_{0}\right\|^{2}+\cdots+\left\|z_{M-1}-H_{M-1} b_{M-1}\right\|^{2}}_{\text {constant, since these terms already deliver the minimum }} \\
& +\left\|z_{M}-\left(H_{M-1}+\varepsilon\right) b_{M}\right\|^{2} .
\end{aligned}
$$

Because of the phase-blindness, the vector $\mathbf{b}$ can be modified such that $b_{M-1}=1$ without loss of generality. Using this fact, and since $H_{M-1}=z_{M-1} b_{M-1}^{*}$ and $H_{M}=H_{M-1}+\varepsilon$ we have

$$
\hat{b}_{M}=\min _{b_{M}}\left\|z_{M}-\left(z_{M-1}+\varepsilon\right) b_{M}\right\|^{2} .
$$


The proof for Theorem 2 establishes a unique solution if $\left|\varepsilon / H_{M}\right|<d_{\min } / 2$. Theorem 3 is thereby proved

\section{Suboptimal Approach For Solving EQ. (14)}

The solution of (14) based on Theorem 1 requires high computational complexity. It is infeasible to compute the global maximum, especially for large memory sizes $M$ and large signal constellations $q$. We first detail how equation (14) can easily be solved based on Theorems 2 and 3 in the noisefree case.

Theorem 3 showed that, in the noise-free case, the channel transfer function can be determined by considering an arbitrary number of adjacent subcarriers $M \geq 2$, if $\mathbf{H}$ exhibits certain features which were detailed in Theorems 2 and 3. In this case, it is trivial to solve equation (14) for $M=2$, since only very few vectors $\mathbf{b}$ exist, namely $q^{2}$ for q-ary symbols. Once the solution for $M=2$ has been obtained, the solution for $M=3$ can easily be determined, since the first two elements of the solution vector $\mathbf{b}$ are the same as those for $M=2$. Hence, for arbitrary $M$, only $q^{2}+(M-2) q$ vectors $\mathbf{b}$ need to be investigated to obtain the optimal solution. The optimal algorithm to solve equation (14), therefore, has complexity $O\left(q^{2}\right)$. Note that an algorithm based on Theorem 2 using exhaustive search has complexity $O(q(M-1))$.

This algorithm can also be applied to the noisy case. However, the algorithm will in general no longer determine the optimal solution vector $\mathbf{b}$, which would be obtained by using exhaustive search or the already described branch-and-bound technique. Instead, a sub-optimal solution will be delivered. Simulation trials have shown that large portions of the vector b can still be obtained correctly. Of course this depends greatly on the noise-variance and the properties of the channel transfer function, in particular the delay spread.

If a-priori knowledge of $\mathbf{b}$ is available, then it is desirable to take advantage of this knowledge for solving equation (14). Based on the considerations above, we propose a slightly modified version of the just described algorithm, which can incorporate a-priori knowledge of $\mathbf{b}$ (if available) as outlined in the following pseudo-code fragment:

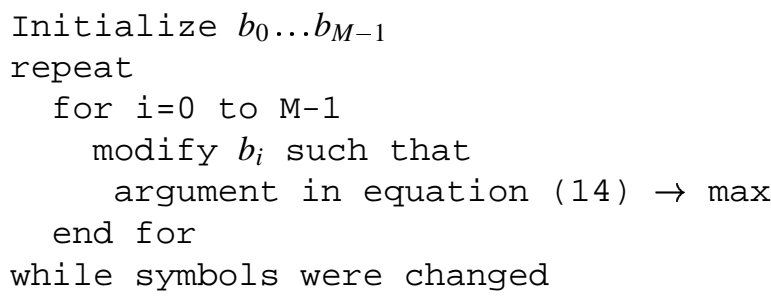

\section{Resolving the Phase Blindness}

In [12], reference symbols are used to overcome the phase blindness. A new method is explored in this section that restores the phase without using reference symbols. Thus our proposed method is totally blind.

The key concept of the proposed method is that two PSKsignal constellations of different order be used within the same OFDM symbol. The two signal constellations are chosen such that the angles between a selected signal point of one
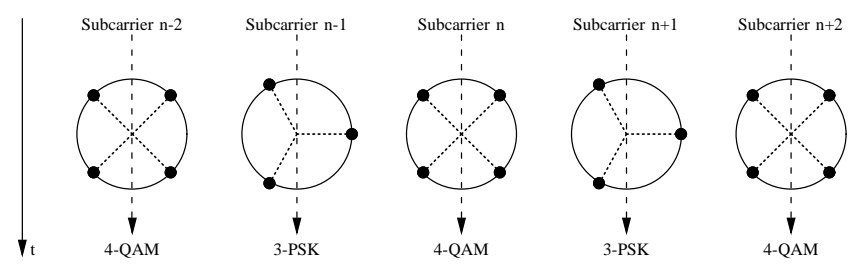

Fig. 2. Modulation schemes on the subcarriers

constellation and any signal point in the other constellation are unique. For example, QPSK and 3-PSK satisfy this property. As shown in Fig. 2, QPSK symbols are interleaved with 3-PSK symbols on alternate OFDM subcarriers. If such a waveform is used, a blind channel estimator based on equation (14) no longer suffers from phase blindness, as we now show.

Let $\hat{\mathbf{b}}$ be a vector solution to equation (14). If only QPSK is used, the vectors $e^{j \varphi \frac{\pi}{2}} \hat{\mathbf{b}}$ with $\varphi=1,2,3$ are also solutions of (14). Likewise, if only 3-PSK is used, the vectors $e^{j \varphi \frac{2 \pi}{3}} \hat{\mathbf{b}}$ with $\varphi=1,2$ are also solutions of (14). However, if both signal constellations are used as described, there is no possibility of phase ambiguity, since the angles of ambiguity in both modulation schemes will not match. In other words, once a vector $\hat{\mathbf{b}}$ solving equation (14) has been found, shifting the phase by $\varphi \frac{\pi}{2}$ would move all ternary symbols away from their possible signal points, and shifting the phase by $\varphi \frac{2 \pi}{3}$ would move all QPSK symbols away from their possible signal points. Thus, (14) has a unique solution.

Other mixtures of signal constellations will also fulfill the above requirement. For example, QPSK can be combined with 5-PSK, and 8-PSK can be combined with 7-PSK or 9PSK. However, if a combination of modulation schemes is used, Theorems 2 and 3 cannot be directly applied. From the above considerations and from Theorem 3 we can derive the following Lemma.

Lemma: By knowing only the received vector $\mathbf{z}$, the channel parameters $\mathbf{H}=\left[H_{0} \cdots H_{M-1}\right]$ and the transmitted symbols $\mathbf{b}=\left[\begin{array}{lll}b_{0} & \cdots & b_{M-1}\end{array}\right]$ are uniquely identifiable up to a complex scaling factor for any $M \geq 2$, if $\left(b_{0}, H_{0}\right) \cdots\left(b_{M-1}, H_{M-1}\right)$ belong to consecutive subcarriers and the channel transfer function coefficients $\mathbf{H}$ change slowly in the frequency domain, i.e. $\left|\frac{\varepsilon}{H_{n}}\right|=\left|\frac{H_{n}-H_{n-1}}{H_{n}}\right|<d_{\min } / 2, n=0, \ldots N-1$., where $d_{\min }=$ $\left|e^{j \frac{2 \pi}{q_{1}}}-e^{j \frac{2 \pi}{q_{2}}}\right|$ with $q_{1}$ and $q_{2}$ denoting the order of the two applied PSK modulation schemes.

Proof: The Lemma follows directly from Theorem 3

Returning to the sub-optimal approach for solving Eq. (14) as it was detailed in the previous section, the combination of modulation schemes introduces a problem. Consider two QPSK and 3-PSK symbols received on adjacent subcarriers as depicted in Fig. 3. The two transmitted symbols have a phase difference of $15^{\circ}$, and the two estimated symbols have a phase
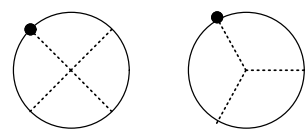

a) transmitted symbols

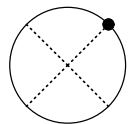

b) estimated symbols
Fig. 3. Possible symbol combinations 
difference of $45^{\circ}$. Our algorithm relies on phase differences. Therefore, the suboptimal algorithm is likely to result in error in the above example, as the phase difference between these two possibilities is only $30^{\circ}$. A constellation with such a small difference will be called an alike looking constellation.

To solve this problem, it is necessary to check if there are any alike looking vectors $\mathbf{b}$ that yield a larger value when used in (14) after a suboptimum has been computed. The simplest approach tries all possible combinations of phase-shifts of the QPSK and 3-PSK symbols:

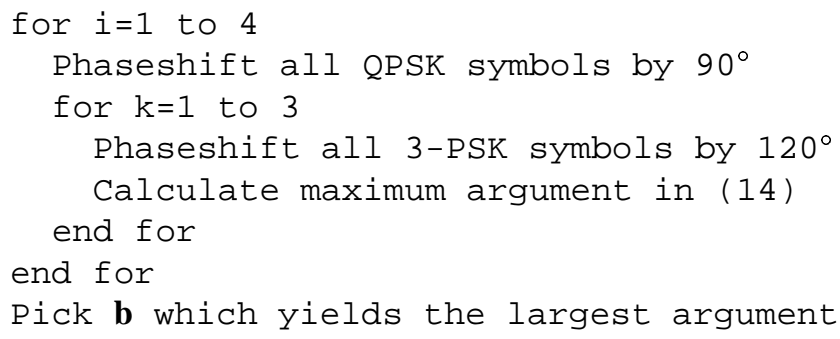

Simulations show that this algorithm almost always catches the global maximum for a COST207 RA channel [16] in the absence of noise. The influence of noise can cause the correct vector $\mathbf{b}$ to be missed. In this case, the performance can be dramatically improved by using a-priori knowledge of the $b_{i}$.

\section{Application OF THE BLIND ChanNEL Estimator AND SIMULATIONS}

\section{A. OFDM Transmitter}

The blind channel estimator was applied to a modified DVB$\mathrm{T}$ system [2]. DVB-T is based on OFDM and uses pilotbased channel estimation for coherent detection of QAMencoded data-symbols ${ }^{2}$. Starting from the $2 \mathrm{k}$-mode with 1705 subcarriers, all pilots were removed, resulting in a system with only 1512 subcarriers and a carrier spacing of $4464 \mathrm{~Hz}$. The regular QAM-modulation scheme was replaced by the combined QPSK/3-PSK scheme. We also investigated the combination of QPSK and 5-PSK. The transmitter of this system is depicted in Fig. 4.

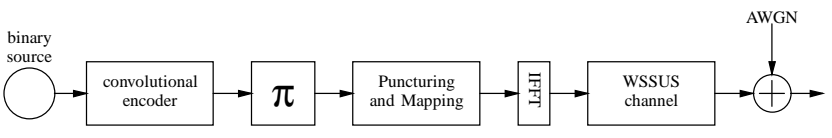

Fig. 4. Transmitter

The bits from the binary source are encoded by a rate$1 / 2$ convolutional coder with generator polynomials $133_{8}$ and $171_{8}$ and bit-interleaved in block $\pi$ according to the DVB$\mathrm{T}$ standard. Code puncturing is used to solve the problem of mapping bits to the 3-PSK and 5-PSK symbols. Fig. 5 shows the trellis of the above mentioned convolutional code. The two coded bits of every second stage in the trellis are left untouched, while the coded bits of the remaining steps are directly converted to ternary symbols according to Table I.

\footnotetext{
${ }^{2}$ DVB-T specifies 4-, 16- or 64-QAM, and hierarchical 16- and 64-QAM. Here we compare our results with 4-QAM
}

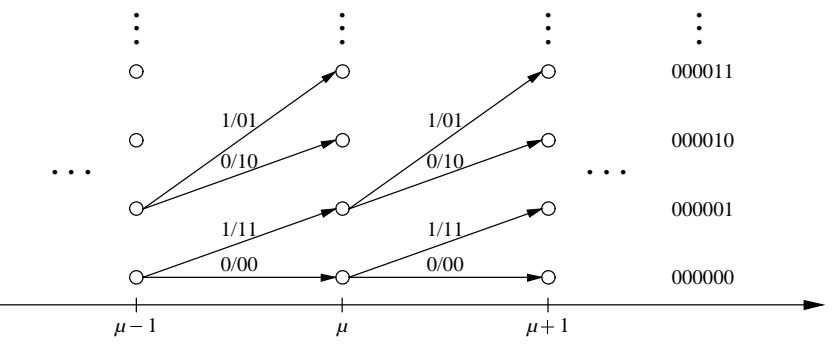

Fig. 5. Trellis of the rate $1 / 2$ convolutional code used in DVB-T systems

When considering the code trellis in Fig. 5, there is only one possible puncturing rule, since the two transitions emerging from each state must be mapped to different 3-PSK symbols.

In general, the distributions of the real- and imaginary parts of the resulting ternary symbols have non-zero mean. Therefore, it is necessary to rotate the above mapping-scheme by $120^{\circ}$ in regular intervals within one OFDM-symbol. This does not affect performance or any of the algorithms and will therefore be disregarded.

The resulting stream of bits and ternary symbols is modulated by the IFFT-block. Attention needs to be paid during the final distribution of the data symbols to the subcarriers, since the QPSK and 3-PSK symbols must alternate.

The feasibility of 5-PSK instead of 3-PSK was explored. A rate- $1 / 3$ code with generator polynomials $133_{8}, 145_{8}$ and $175_{8}$ was chosen as basis code. The even-numbered trellis steps are punctured by simply dropping the parity bit of the second generator polynomial. The odd-numbered trellis steps are mapped to 5-PSK symbols according to Table II. In contrast to the 3-PSK case, the optimal puncturing scheme is non-obvious. A heuristic approach was taken by mapping any two transitions emerging from a state to signal points which are at a maximum distance from each other, e.g., 0 and 2, similar to mapping by set partitioning. As with 3-PSK, the mapping scheme needs to be rotated regularly to avoid dc offsets, this time by $72^{\circ}$.

TABLE I

CONVERSION OF CODED BITS TO 3-PSK SYMBOL. The NUMBERS 0,1 AND 2 REPRESENT THE DIFFERENT SIGNAL POINTS OF A 3-PSK SYMBOL.

\begin{tabular}{|c|c||c|c|}
\hline coded bits & ternary symbol & coded bits & ternary symbol \\
\hline 00 & 0 & 01 & 2 \\
11 & 1 & 10 & 1 \\
\hline
\end{tabular}

TABLE II

CONVERSiOn OF CODED BITS TO 5-PSK SYMbOL. THE NUMBers $0,1,2$, 3 AND 4 CORRESPOND TO THE DIFFERENT SIGNAL POINTS OF A 5-PSK SYMBOL.

\begin{tabular}{|c|c||c|c|}
\hline coded bits & quintary symbol & coded bits & quintary symbol \\
\hline 000 & 0 & 100 & 0 \\
001 & 1 & 101 & 4 \\
010 & 2 & 110 & 3 \\
011 & 3 & 111 & 2 \\
\hline
\end{tabular}




\section{B. Soft-values for arbitrary q-PSK symbol constellations}

The receiver should be able to soft-decode the received data stream. When using a Viterbi algorithm [4] or a MAP algorithm [17] to soft-decode the received data symbols, each coded bit is assigned a soft-value $L_{c}$, and each decoded information bit is assigned a soft-value $L_{i}$. Recall the definition of the channel soft-values $L^{c h}$ for bit $c$ of a received symbol $y$ (see for example [17]):

$$
L^{c h}(c)=\ln \frac{P(c=1 \mid y)}{P(c=0 \mid y)}=\ln \left(\frac{P(y \mid c=1)}{P(y \mid c=0)} \cdot \frac{P(c=1)}{P(c=0)}\right) \text {. }
$$

In the pure QPSK-case, the MAP-decoder determines the likelihood for every state transition in the trellis to produce the output soft-value sets $L_{c}$ and $L_{i}$ for the code bits and information bits, respectively. The likelihood of a state transition is calculated from the two soft-values $L^{c h}$ belonging to a particular state transition, and the state probabilities of the originating and terminating state.

It is well known how to determine the soft-values for the coded bits of QPSK-symbols (compare [4]). While it is also possible to extract soft-values for the two or three code bits which were punctured to form a 3- resp. 5-PSK-symbol, we will extend the above definition of soft-values to q-ary symbols. This allows us to directly assign a soft-value to a transition within the code trellis without first determining the soft-values for the code bits.

To extend this concept to q-ary symbols, a set of $q$ softvalues is introduced, each indicating the likelihood that one of the $q$ signal points was received. That is, for every received q-ary symbol, $q$ soft-values $L_{s}^{c h}(x)$ will be calculated, with $s$ being the different signal points of the q-ary symbol. The definition of $L_{s}^{c h}(x)$ is analog to the definition of soft-values for bits in Eq. (20):

$$
\begin{aligned}
& L_{s}^{c h}(x)=\ln \frac{P(x=s \mid y)}{P(x \neq s \mid y)}=\ln \left(\frac{P(y \mid x=s)}{P(y \mid x \neq s)} \cdot \frac{P(x=s)}{P(x \neq s)}\right), \\
& s=0 \ldots q-1,
\end{aligned}
$$

with $x$ being the transmitted symbol.

Using this soft-output definition, it is easy to modify a MAP-algorithm (see for example [17], [18]) to soft-decode a mixed stream of bits and ternary/quintary symbols. The likelihood for a state transition will either be influenced by two soft-values $L^{\text {ch }}$ of a state transition associated with a QPSKsymbol, or by one soft-value from the set $L_{s}^{c h}$ for any state transition associated with a 3-PSK/5-PSK-symbol

\section{Receiver}

The basic structure of the receiver is shown in Fig. 6, where the discrete time index $l$ is introduced. The core of the receiver is an OFDM-symbol buffer, which holds the last nine received OFDM-symbols $\mathbf{z}_{\mathbf{l}}$, together with their associated channel transfer functions $\mathbf{H}_{\mathbf{l}}$ and the estimated symbols $\mathbf{b}_{\mathbf{l}}$. The number of stored OFDM-symbols can be varied, but nine was chosen to match the order of the Wiener Filter in the time direction of the pilot-based reference system (see section V-E). The OFDM-symbol buffer allows for signal processing of the stored vectors $\mathbf{H}_{\mathbf{l}}$. In particular, the $\mathbf{H}_{\mathbf{l}}$ can be low-pass filtered

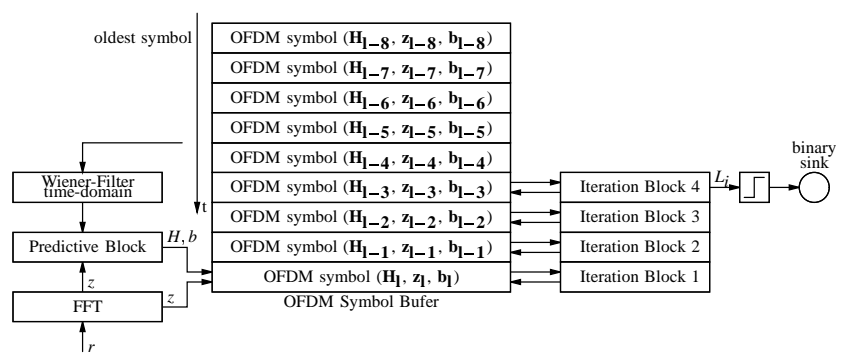

Fig. 6. Structure of OFDM symbol buffer

using two Wiener-Filters, one in the time domain and one in the frequency domain. The filter coefficients are based on the Wiener design criterion [19], where the filters in the time and frequency domains were designed for a maximum Doppler shift of $f_{d, \max }=200 \mathrm{~Hz}$ and a maximum channel delay spread of $\tau_{m}=10 \mu$ s respectively.

A newly received OFDM-symbol is demodulated by the FFT-block and stored in the OFDM-symbol buffer. The channel transfer function $\mathbf{H}_{\mathbf{l}}$ for this OFDM-symbol is then estimated by Iteration Block 1 having the internal structure shown in Fig. 7. The suboptimal (blind) channel estimator inside this block runs the algorithm introduced in Sect. III multiple times on blocks of $M$ OFDM subcarriers to estimate the channel transfer function $\mathbf{H}_{\mathbf{l}}^{\prime}$ of one OFDM-symbol. The vector $\mathbf{b}_{\mathbf{l}}$ serves as a-priori knowledge to the blind estimator. The estimated channel coefficients $H_{l, k}^{\prime}$ and the received symbols $z_{l, k}$ are fed into the demapper, which provides soft-values on the coded bits $\left(L^{c h}\right)$ and ternary/quintary symbols $\left(L_{s}^{c h}\right)$. These soft-values are deinterleaved in block $\pi^{-1}$. The subsequent inner convolutional decoder utilizes redundancy and improves estimation results. The decoder outputs soft-values on the information bits, $L_{i}$, and code bits, $L_{c}$; hard-decisions are made on the code bits, which are interleaved and mapped to ternary/quintary symbols.

The estimated transfer function $\mathbf{H}_{\mathbf{l}}^{\prime \prime}$ is Wiener filtered to reduce noise and even out estimation errors. The results of Iteration Block 1 are stored in the OFDM symbol buffer.

The described process is iterated during the following OFDM time-steps by multiple instances of the iteration block. The blind estimator is only active in Iteration Block 1 and disabled within the other iteration blocks, which has proven to improve estimation results during our simulation runs.

A-priori knowledge is provided to the blind estimator of Iteration Block 1 by predicting the transfer function for the latest OFDM-symbol with a Wiener-Filter. This Wiener-

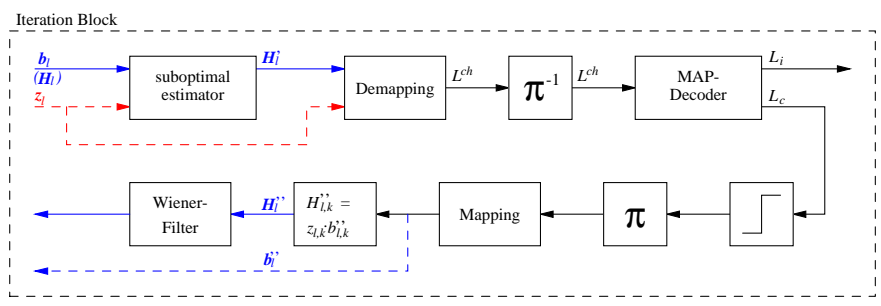

Fig. 7. Block diagram for one step of the iteration 
Filter is applied in the time-direction and was designed for a maximum Doppler-Frequency of $200 \mathrm{~Hz}$ [20]. The predicted transfer function is fed into an instance of the Iteration Block, which will be called the Predictive Block. Since the blind estimator can only utilize the $b_{i}$ as $a$-priori knowledge, it needs to be disabled in the Predictive Block. The new $b_{i}$ produced by the Predictive Block serve as a-priori knowledge to the blind estimator within Iteration Block 1.

This receiver design delivers good performance and also allows for an efficient implementation in hardware, since all iterations on the different OFDM-symbols can be performed in parallel. However, there are still ways to further increase performance. One way is to add the soft-values produced by the inner decoder of the Predictive Block and those produced by the inner decoder of Iteration Block 1 just before the hard decision inside Iteration Block 1. This improves performance since there are some OFDM-symbols for which the blind estimator produces rather bad or even useless results, e.g. when the channel is in a fade ${ }^{3}$. In these cases, good channel estimation might still be possible with a predictive WienerFilter.

A second possibility is to use the Wiener-Filter in timedirection to filter the transfer function of each OFDM-symbol stored in the buffer before each iteration. This improves the channel estimates for OFDM-symbols received during a channel fade, when newly received symbols start delivering good estimation results again.

Decoding multiple OFDM-symbols at the same time also nicely solves the problem of initializing the values of the backward recursion in the MAP-algorithm of the inner decoder [18]. For all but the latest received symbol, the backwardrecursion can be initialized by taking the end-values of the backward-recursion of the subsequent OFDM-symbol.

\section{Channel Model and Simulation Environment}

Our simulations used the COST207 RA (Rural Area), TU (Typical Urban) and BU (Bad Urban) channels having a maximum delay spread of $0.7 \mu \mathrm{s}, 7 \mu \mathrm{s}$ and $10 \mu \mathrm{s}$, respectively [16]. The usage of these models was motivated by a number of simulation studies that have been carried out with these models (e.g. [1], [21]). The guard interval of the OFDM-system was chosen to be a quarter of an OFDM-symbol length, yielding an OFDM-symbol duration of $280 \mu$ s. A shorter guard interval would be sufficient for any of the investigated channels, but we chose the guard interval duration to match the pilot-based reference system (see Sect. V-E).

Simulations were performed at a Doppler frequency of $f_{d}=$ $193 \mathrm{~Hz}$, which corresponds to a high vehicular speed of about $200 \mathrm{~km} / \mathrm{h}$ at a typical carrier frequency of $1 \mathrm{GHz}$. This shows the feasibility of the proposed algorithm in a rapidly timevariant mobile environment.

The WSSUS-channels were simulated according to the model introduced in [22], which describes the channel's time-

\footnotetext{
${ }^{3}$ Note that interleaving does not help the blind channel estimator since it needs to consider adjacent subcarriers.
}

variant impulse response as

$$
h(\tau, t)=\lim _{\mu \rightarrow \infty} \frac{1}{\sqrt{\mu}} \sum_{m=1}^{\mu} e^{j \theta_{m}} e^{j 2 \pi f_{D_{m}} t} \delta\left(\tau-\tau_{m}\right) .
$$

The Fourier-Transform of equation (22) with respect to $\tau$ yields the channel's time-variant frequency response:

$$
H(f, t)=\lim _{\mu \rightarrow \infty} \frac{1}{\sqrt{\mu}} \sum_{m=1}^{\mu} e^{j \theta_{m}} e^{j 2 \pi f_{D_{m}} t} e^{-j 2 \pi f \tau_{m}} .
$$

For each of the $\mu$ paths, the phase-shift $\theta_{m}$, the Dopplershift $f_{D_{m}}$ and the delay $\tau_{m}$ are randomly chosen from the corresponding probability density function $p_{\theta}(\theta), p_{f_{D}}\left(f_{D}\right)$ or $p_{\tau}(\tau)$ of the channel model [22]. For the simulations, the number of paths was chosen to be $\mu=50$, which is a good tradeoff between simulation speed and accuracy. Note that in this model, the continuous power-delay profiles from COST207 are approximated with $\mu$ rays, in contrast to the also often used 6- or 12-ray models.

\section{E. Pilot-Based Reference System}

The pilot-based DVB-T system with an adaptive receiver presented in [15] was used as a reference system. The receiver employs an adaptive Wiener-Filter and achieves good channel estimates without any iterations. The regular DVB-T pilot pattern with an overhead of $12.8 \%$ was employed, which accommodates channels with a delay spread of up to $56 \mu \mathrm{s}$ [2].

\section{F. BER Results}

Figs. 8 and 9 show the simulation results for the RA (Rural Area), TU (Typical Urban) and BU (Bad Urban) channel models, respectively. The BER of the proposed receiver design with QPSK/3-PSK and QPSK/5-PSK is plotted along with BER-curves of the pilot-based DVB-T reference system. For comparison, the BER-results of the blind channel estimator with only one iteration and QPSK/5-PSK are plotted for the RA and TU channels.

Measurements were started after the $20^{\text {th }}$ OFDM-symbol was received. For most scenarios, these 20 symbols are enough to achieve convergence, except for some situations with a low $E_{b} / N_{0}$. This correspond to a delay of only $5.6 \mathrm{~ms}$, which is

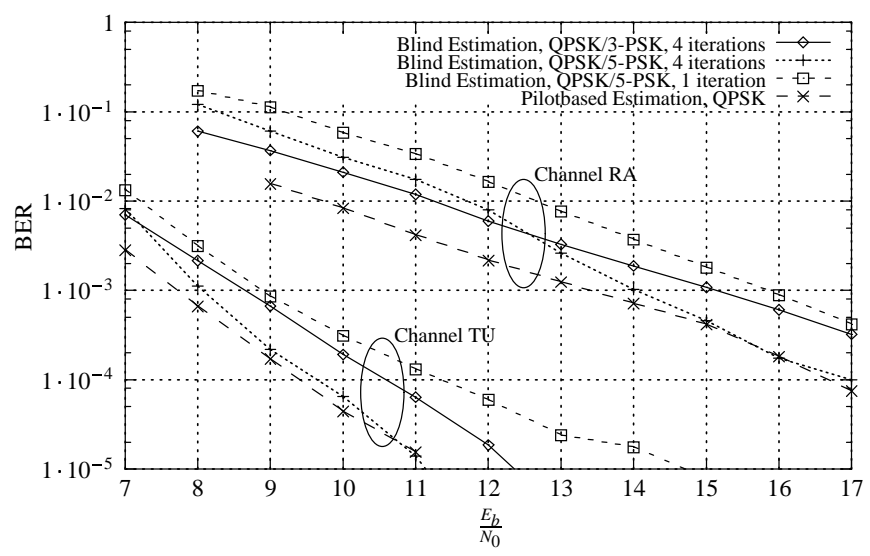

Fig. 8. BER for RA and TU channels, $f_{D, \max }=193 \mathrm{~Hz}$, block length $M=10$ 


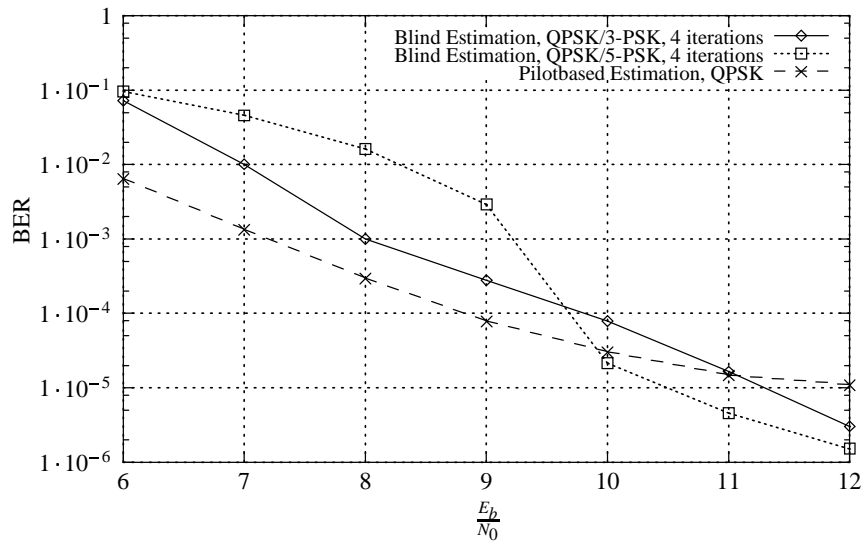

Fig. 9. BER for BU channel, $f_{D, \max }=193 \mathrm{~Hz}$, block length $M=10$

acceptable for a broadcasting system. Delaying the beginning of the BER measurements by 20 symbols will allow us to separate BER-performance from convergence behavior, which is detailed in the next section.

The QPSK/5-PSK scheme provides stronger coding than the QPSK/3-PSK scheme. On the other hand, it is more difficult to recover the phase for QPSK/5-PSK, since the angular difference between signal points is smaller. At a low $E_{b} / N_{0}$, the ability of the blind estimator to determine the channel transfer function has a strong influence on BER performance, which is why QPSK/3-PSK performs better. For higher $E_{b} / N_{0}$, the blind estimation results with QPSK/5-PSK become better and approach that of QPSK/3-PSK. Hence, the stronger coding becomes the dominating performance factor. Consequently, QPSK/3-PSK obtains better BER-performance at low $E_{b} / N_{0}$, whereas QPSK/5-PSK performs better at higher $E_{b} / N_{0}$.

In any case, the achievable BER-performance with both modulation schemes lies within $2 \mathrm{~dB}$ of the pilot-based reference system. At reasonable BER-values of around $10^{-4}$, the BER performance of the blind system is comparable to that of the reference system.

The effect of using one instead of four iteration blocks is demonstrated for QPSK/3-PSK on RA and TU channels. As expected, performance improves with more iterations. The

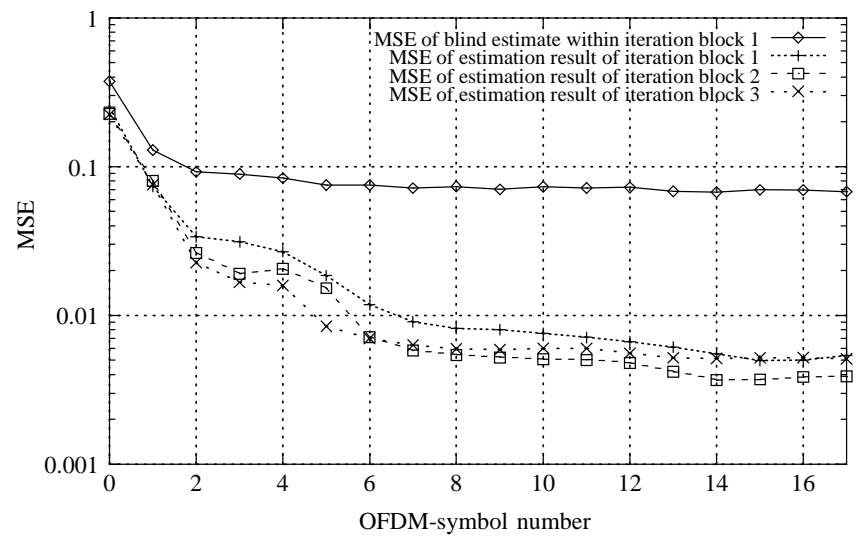

Fig. 10. Convergence behavior: RA channel, $E_{b} / N_{0}=12 \mathrm{~dB}$, block length $M=10$, QPSK $/ 3-P S K$ influence of the block size $M$ within the blind estimator is less obvious. As mentioned previously, larger values of $M$ alleviate the effect of noise. On the other hand, a fade within a block of length $M$ might spoil the blind estimate of that particular block. Therefore, it is desirable to partition an OFDM-symbol into as many blocks as possible. For all of our results, we chose a value of $M=10$, which proved to be a good compromise.

The missing pilot symbols of the blind system compared to the pilot-based reference system directly translates to an increased spectral efficiency. Even though different modulation schemes are used, the same number of information bits are transmitted per OFDM-symbol. Disregarding the necessary guard bands at both sides of the OFDM signal spectrum, the number of information bits transmitted per unit bandwidth is increased by $12.8 \%$.

\section{G. Convergence Rate}

To investigate the convergence behavior of the algorithm, a discontinuity in the channel impulse response was introduced by setting all taps to zero. Figs. 10 through 15 plot the MSE of the channel estimate as a function of the OFDM-symbol received after the discontinuity, where the OFDM-symbol with index 0 is received over the regular mobile channel. This mobile channel was simulated with the assumption of an unchanged channel transfer function for the duration of one OFDM-symbol, i.e., no ICI. This assumption was necessary to provide a unique channel transfer function for each OFDM-symbol so that the MSE can be computed, while still maintaining mobility. The maximum Doppler-frequency was $f_{D_{\max }}=193 \mathrm{~Hz}$. The $E_{b} / N_{0}$ was $12 \mathrm{~dB}$ for the RA channel and $10 \mathrm{~dB}$ for the $\mathrm{TU}$ and $\mathrm{BU}$ channels.

As expected, the convergence behavior deteriorates as the delay spread of the channel increases. Observe that the algorithm converges faster for QPSK/3-PSK as compared to QPSK/5-PSK, while the BER-performance is better for QPSK/5-PSK. Note that the BER performance improves dramatically with increased iterations, even though there is little change in the convergence rate. The difference in convergence rate is minor for the RA and TU channels, but is significant for the BU channel. The BU channel with QPSK/5-PSK requires

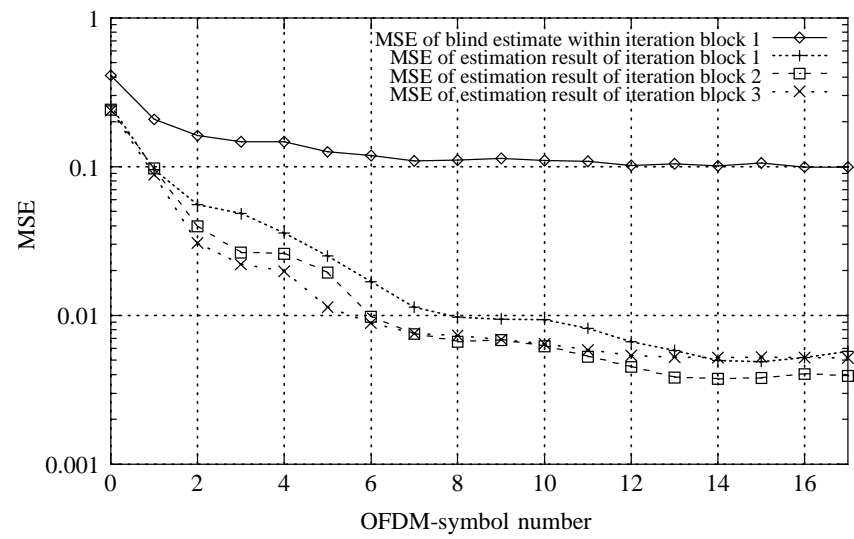

Fig. 11. Convergence behavior: RA channel, $E_{b} / N_{0}=12 \mathrm{~dB}$, block length $M=10, \mathrm{QPSK} / 5-\mathrm{PSK}$ 


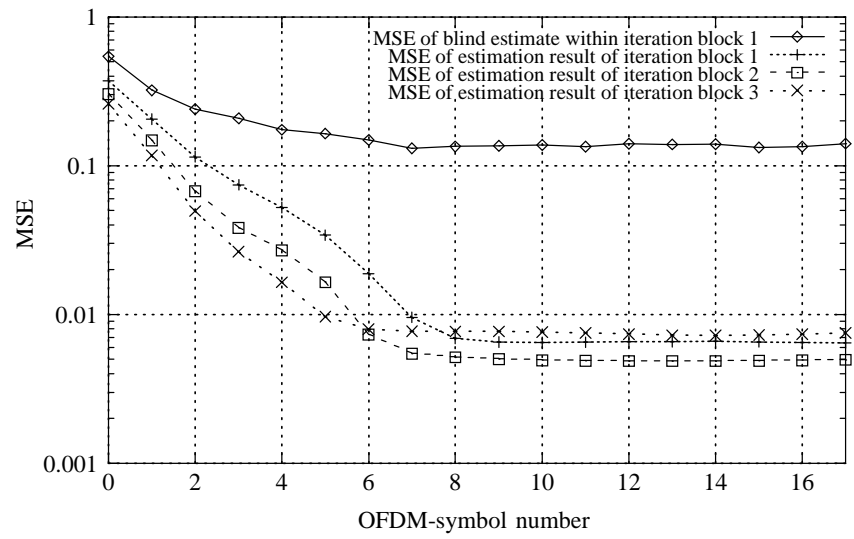

Fig. 12. Convergence behavior: TU channel, $E_{b} / N_{0}=10 \mathrm{~dB}$, block length $M=10, \mathrm{QPSK} / 3-\mathrm{PSK}$

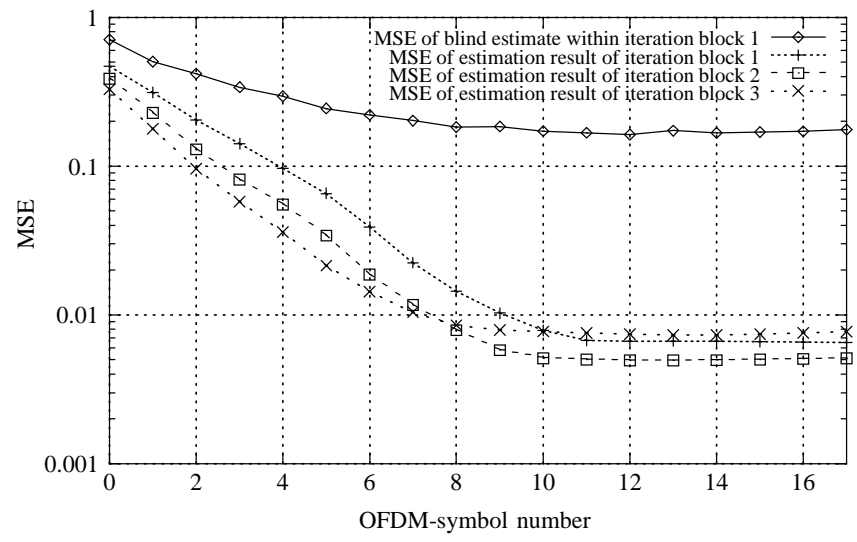

Fig. 13. Convergence behavior: TU channel, $E_{b} / N_{0}=10 \mathrm{~dB}$, block length $M=10, \mathrm{QPSK} / 5-\mathrm{PSK}$

the longest convergence time. Simulations showed that this difference becomes larger as $E_{b} / N_{0}$ decreases. Therefore, the overall system design involves a tradeoff between convergence speed and BER performance.

\section{H. Complexity Analysis}

Referring to Sect. III, the sub-optimal algorithm for solving Eq. (14) can be realized with complexity $O\left(q^{2}\right)$. This is a very small value and also holds if modulation schemes are combined. In the latter case, the $q$ of the higher order modulation scheme determines the complexity. Hence, the central part of the receiver, which is the blind channel estimator itself, consumes a small fraction of the computational resources required to implement the receiver. The remainder of the receiver is no more complex than a well-known turbo decoder, which makes several (de-)interleaving and de/encoding steps. It is obvious that these turbo-like principles can be easily applied to the proposed receiver by concentrating the iterations in a turbo decoder. The complexity of the presented channel estimation approach is, therefore, quite manageable. We believe that our blind channel estimator has low complexity when compared to other blind channel estimation approaches, especially those based on statistics.

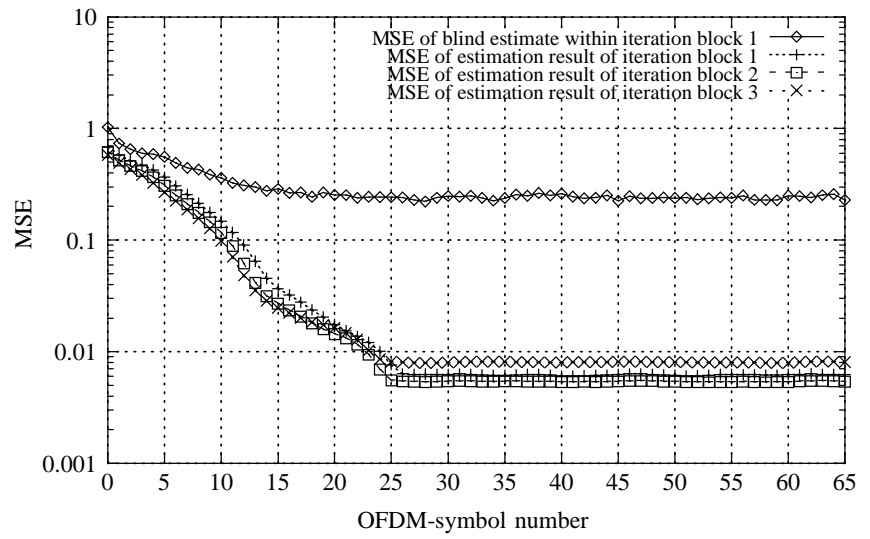

Fig. 14. Convergence behavior: BU channel, $E_{b} / N_{0}=10 \mathrm{~dB}$, block length $M=10$, QPSK/3-PSK

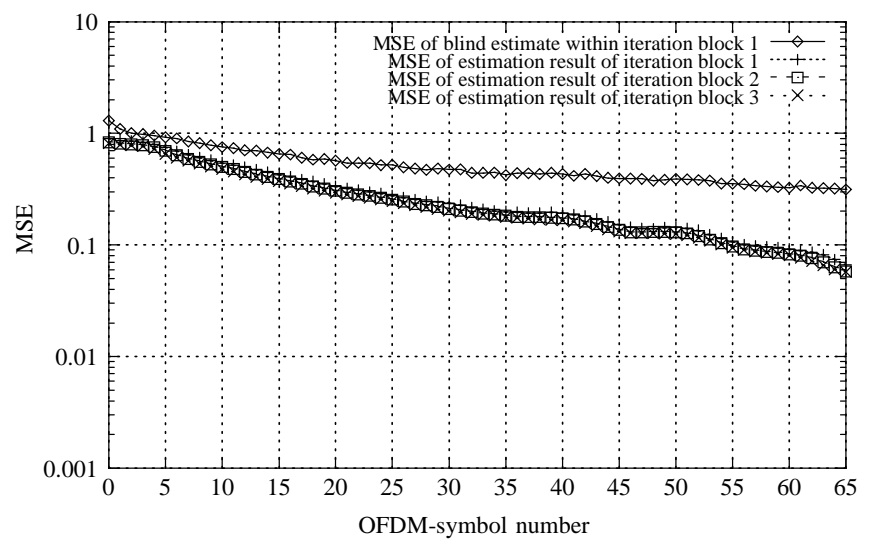

Fig. 15. Convergence behavior: BU channel, $E_{b} / N_{0}=10 \mathrm{~dB}$, block length $M=10$, QPSK/5-PSK

\section{Broadcasting Environments and OFDM Bursts}

All simulation results were determined in a broadcasting environment with a continuous stream of OFDM-symbols. If only data-bursts are transmitted, the receiver needs to be adapted in order to deliver optimum performance. Depending on the assumed worst case channel and the tolerable delay within the receiver, it might be necessary to transmit reference symbols within the first OFDM-symbol of the burst. This would allow the receiver to quickly pick up the channel and might be necessary, especially for the BU channel where slow convergence is observed.

On the other hand, the fast convergence behavior with the TU channel, and especially RA channel, makes it possible to get by without reference symbols for these channels. Since the MSE of the channel estimate already drops below $10 \%$ after a couple of received symbols, the receiver will be able to deliver a good estimate of the channel transfer function for a large portion of the OFDM-burst. The receiver will then be able to provide a-priori knowledge of the channel transfer function for the first few OFDM-symbols by Wiener-Filtering in the time-direction. This in turn will allow blind channel estimation as we have described.

The MSE-measurements show that this procedure is feasible with bursts of several OFDM-symbols (e.g. 10-15 OFDM- 
symbols, corresponding to $2.8-4.2 \mathrm{~ms}$ duration). A delay of several milliseconds is comparable to that of state-of-the-art mobile communication systems (such as UMTS) and is quite acceptable for real-time data- and voice-applications.

\section{CONCLUSION}

A novel blind channel estimation scheme was presented. In contrast to most other blind channel estimation approaches, it uses no second or higher order statistics. It features relatively low complexity and a very fast convergence rate. By applying a combination of modulation schemes, the absolute phase of the channel transfer function can be resolved without the need for reference symbols, making the algorithm, we believe, to be the first fast converging truly blind channel estimation algorithm. Compared to a pilot-based system, the spectral efficiency is significantly increased while maintaining a competitive BER-performance. Simulations were performed for a modified DVB-T system. The results clearly indicate the feasibility of the proposed approach even if data bursts are transmitted. Finally, the proposed approach maximizes the spectral efficiency by avoiding any reference symbols or pilots, while improving the $E_{b} / N_{0}$ performance by using a coherent detection rather than differential detection.

\section{APPENDIX}

In this Appendix, we estimate the maximum tolerable channel delay such that Theorems 1 and 2 and the Lemma are valid.

From the channel model introduced in Sect. V-D, observe from Eq. (23) that the real and imaginary part of the time-variant frequency response $H(f, t)$ is a superposition of an infinite number of phase-shifted and scaled complex oscillations:

$$
H(f, t)=\lim _{\mu \rightarrow \infty} \frac{1}{\sqrt{\mu}} \sum_{m=1}^{\mu} \underbrace{e_{\text {complex oscillation }}^{j \theta_{m}} e^{j 2 \pi f_{D_{m}} t}}_{\text {phaseshift }} \underbrace{e^{-j 2 \pi f \tau_{m}}} .
$$

We will use the complex oscillation with the largest angular frequency to estimate the $\varepsilon$ from the presented theorems, which can be tolerated by a particular channel.

From system theory it is well known that the Fourier transform of a delta function is a complex valued exponential function. Applied to equations (22) and (23) we get:

$$
\delta\left(\tau-\tau_{m}\right) \Longleftrightarrow 2 \pi e^{-j 2 \pi f \tau_{m}}
$$

Hence, the maximum delay of the mobile channel directly translates to the complex oscillation with the largest angular frequency within the channel frequency response $H(f, t)$. To estimate $\varepsilon$, we will only consider the oscillation term $g(f)$ with the largest angular frequency in $H(f, t)$ :

$$
g(f)=K e^{-j 2 \pi f \tau_{m}},
$$

where $K$ is a constant complex scaling factor depending on the channel gain. Taking into account the carrier spacing of $4464 \mathrm{~Hz}$ (for the DVB-T system) and by utilizing the subcarrier index $k$ we get:

$$
g(f)=K e^{-j 2 \pi 4464 \frac{1}{s} k \tau_{m}}=K e^{-j 2 \pi 4464 \frac{1}{s} k \tau_{m}} \approx K e^{-j 28048 \frac{1}{s} k \tau_{m}} .
$$

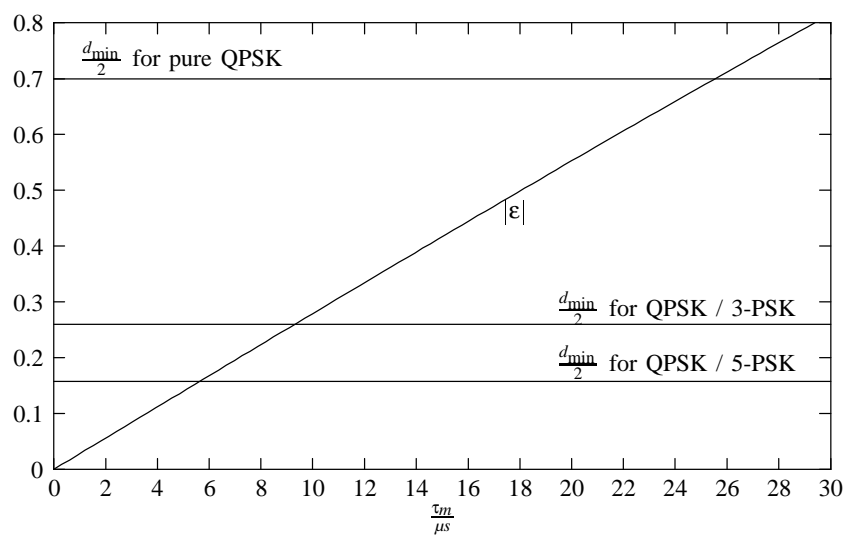

Fig. 16. Estimate of $\varepsilon_{\max }$ against the maximum channel delay $\tau_{m}$.

Finally, we get the estimate for the maximum value of $\left|H_{k}-H_{k+1}\right|$ as

$$
|\varepsilon|=K\left(1-K e^{-j 28048 \frac{1}{s} \tau_{m}}\right) .
$$

$\left|\varepsilon_{\max }\right|$ is plotted against the maximum channel delay $\tau_{m}$ in Fig. 16 for $K=1$. In addition, the graph contains $\frac{d_{\min }}{2}$ according to Theorem 2 for the case of QPSK only, and $\varepsilon$ according to the Lemma for the case of combined QPSK/3-PSK and QPSK/5-PSK. Note that $K$ was chosen to be 1 because this is the mean value of $|H(f, t)|$.

Observe that the condition $\frac{d_{\min }}{2}<|\varepsilon / K|$ resp. $\frac{d_{\min }}{2}<|\varepsilon|$ as introduced in Theorem 2 is fulfilled even for large maximum channel delays $\tau_{m}>20 \mu s$. We can conclude that, if only QPSK is used, blind channel estimation according to Theorems 2 and 3 is possible even for channels having a large maximum delay, such as COST207 Hilly Terrain (HT) channel [16]. Note that in the case of pure QPSK the channel estimate exhibits a phase ambiguity and, therefore, reference symbols are needed to resolve this ambiguity.

The condition $\frac{d_{\min }}{2}<|\varepsilon|$ according to the Lemma is fulfilled for short to medium maximum channel delays. The COST207 RA channel [16] with $\tau_{m}=0.7 \mu s$ poses no problem for either QPSK/3-PSK or QPSK/5-PSK. The COST207 TU channel [16] with $\tau_{m}=7 \mu \mathrm{s}$ is more problematic. For QPSK/3-PSK, the condition is still fulfilled. However, for For QPSK/5-PSK, the Lemma no longer holds. However, simulations showed that blind channel estimation is still possible since only a small portion of the received energy is received with a path delay of $6 \mu \mathrm{s}$ or more.

The most problematic channel is the COST207 BU channel [16] with $\tau_{m}=10 \mu s$. For QPSK/3-PSK, the condition is almost fulfilled, but in the QPSK/5-PSK does not hold. This is supported by the simulations, which show convergence for the QPSK/3-PSK-case (Fig. 14), but a problematic convergence behavior for the QPSK/5-PSK-case (Fig. 15).

\section{ACKNOWLEDGEMENTS}

This work was financially supported by the Information Technology Society of the Association for Electrical Electronic \& Information Technologies, Germany (VDE) and was supported in part by the National Science Foundation under 
Grant CCR-0121565. The original DVB-T simulation system was developed by Frieder Sanzi at the Institute of Telecommunications, University of Stuttgart, Germany.

\section{REFERENCES}

[1] R. Burow, K. Fazel, P. Höher, O. Klank, H. Kussmann, P. Pogrzeba, P. Robertson, M.J. Ruf: 'On the Performance of the DVB-T System in Mobile Environments', in Proc. IEEE Globecom '98, Vol. 4, pp. 2198 2204, Nov. 1998

[2] 'Digital Video Broadcasting (DVB); Framing structure, channel coding and modulation for digital terrestrial television (DVB-T)', European Telecommunication Standard, ETS 300 744, March 1997

[3] 'Radio broadcasting systems; Digital Audio Broadcasting (DAB) to mobile, portable and fixed receivers', European Telecommunication Standard, ETS 300 401, May 1997

[4] J. G. Proakis: 'Digital Communications', McGraw-Hill, 1995

[5] B. Muquet, M. de Courville: 'Blind and Semi-Blind Channel Identification Methods using Second Order Statistics for OFDM Systems', in Proc. IEEE ICASSP '99, Vol. 5, pp. 2745-2748, March 1999

[6] F.-C. Zheng, S. McLaughlin, B. Mulgrew: 'Blind equalization of nonminimum phase channels: higher order cumulant based algorithm', IEEE Transactions on Signal Processing, Vol. 41, No. 2, pp. 681 - 691, Feb. 1993

[7] S. Chen, S. McLaughlin: 'Blind channel identification based on higherorder cumulant fitting using genetic algorithms', in Proc. IEEE Signal Processing Workshop on Higher-Order Statistics, pp. 184-188, July 1997

[8] H. H. Zeng, L. Tong: 'Blind Channel Estimation Using the Second-Order Statistics: Asymptotic Performance and Limitations', IEEE Transactions on Signal Processing, Vol. 45, No. 8, pp. 2060-2071, August 1997

[9] B. Muquet, M. de Courville, P. Duhamel: 'Subspace-Based Blind and Semi-Blind Channel Estimation for OFDM Systems', IEEE Transactions on Signal Processing, Vol. 50, pp. 1699-1712, July 2002

[10] U. Tureli, H. Liu: 'Blind Carrier Synchronization and Channel Identification for OFDM Communications', in Proc. IEEE ICASSP '98, Vol. 6, pp. 3509-3512, May 1998

[11] T. Petermann, S. Vogeler, K-D. Kammeyer, D. Boss: 'Blind Turbo Channel Estimation in OFDM Receivers', in Proc. $35^{\text {th }}$ Asilomar Conference on Signals, Systems and Computers, Vol. 2, pp. 1489 -1493, November 2001

[12] N. Chotikakamthorn, H. Suzuki: 'On Identifiability of OFDM Blind Channel Estimation', in Proc. VTC 1999 - Fall, Amsterdam, Netherlands, Vol. 4, pp. 2358-2361, Sept. 1999

[13] W. L. Winston: 'Introduction to Mathematical Programming: Applications and Algorithms', $2^{\text {nd }}$ edition, Duxbury Press, 1995

[14] G. L. Stüber: 'Principles of Mobile Communication', 2 ${ }^{\text {nd }}$ Edition, Kluwer Academic Publishers, 2001

[15] M. Necker, F. Sanzi, J. Speidel: 'An Adaptive Wiener-Filter for Improved Channel Estimation in mobile OFDM-Systems', in Proc. IEEE ISSPIT 2001, pp. 213-216, Dec. 2001

[16] Cost 207 WG1, 'Proposal on Channel Transfer Functions to be used in GSM Tests late 1986', CEPT, Paris, September 1986

[17] L. R. Bahl, J. Cocke, F. Jelinek and J. Raviv: 'Optimal Decoding of Linear Codes for Minimizing Symbol Error Rate', IEEE Transactions on Information Theory, Vol. 20, No. 20, pp. 284-287, pp. 284-287, March 1974

[18] J. Hagenauer, E. Offer and L. Papke: 'Iterative Decoding of Binary Block and Convolutional Codes', IEEE Transactions on Information Theory, Vol. 42, No. 2, pp. 429-445, March 1996

[19] P. Höher, S. Kaiser, P. Robertson: 'Two-Dimensional Pilot-SymbolAided Channel Estimation by Wiener Filtering', in Proc. of IEEE ICASSP '97, Vol. 3, pp. 1845-1848, April 1997
[20] P. Hoeher, S. Kaiser and P. Robertson: 'Pilot-symbol-aided channel estimation in time and frequency', in Proceedings of the Sixth Communication Theory Mini-Conference in conjunction with IEEE GLOBECOM '97, pp. 90-96, Nov. 1997

[21] H. Kußmann, M. Ruf: 'Die Leistungsfähigkeit des DMB- und DMB$\mathrm{T}$ Systems in einer mobilen Empfangssituation', in Der Fernmelde Ingenieur, year 52, Nov./Dec. 1998

[22] P. Hoeher: 'A Statistical Discrete-Time Model for the WSSUS Multipath Channel', IEEE Transactions on Vehicular Technology, Vol. 41, No. 4, pp. 461-468, Nov. 1992

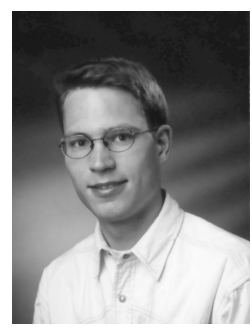

Marc Necker received the M.S. degree from the Georgia Institute of Technology in 2001 and the Diploma degree in Electrical Engineering and Information Technology from the University of Stuttgart, Germany, in 2002. He is currently a member of the scientific staff at the Institute of Communication Networks and Computer Engineering at the University of Stuttgart where he is working towards his Ph.D.

His research interests are in wireless communications, channel estimation for OFDM systems and modeling and architecture of radio access networks.

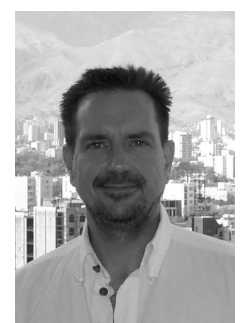

Dr. Gordon L. Stüber received the B.A.Sc. and Ph.D. degrees in Electrical Engineering from the University of Waterloo, Ontario, Canada, in 1982 and 1986 respectively. Since 1986, he has been with the School of Electrical and Computer Engineering, Georgia Institute of Technology, where he is currently the Joseph M. Pettit Professor in Communications.

Dr. Stüber's research interests are in wireless communications and communication signal processing. $\mathrm{He}$ is author of the textbook Principles of Mobile Communication, Kluwer Academic Publishers, 1996, 2/e 2001. He was co-recipient of the Jack Neubauer Memorial Award in 1997 recognizing the best systems paper published in the IEEE Transactions on Vehicular Technology. He became an IEEE Fellow in 1999 for contributions to mobile radio and spread spectrum communications. He received the IEEE Vehicular Technology Society James R. Evans Avant Garde Award in 2003 recognizing his contributions to theoretical research in wireless communications.

Dr. Stüber served as Technical Program Chair for the 1996 IEEE Vehicular Technology Conference (VTC'96), Technical Program Chair for the 1998 IEEE International Conference on Communications (ICC'98), General Chair of the Fifth IEEE Workshop on Multimedia, Multiaccess and Teletraffic for Wireless Communications (MMT'2000), General Chair of the 2002 IEEE Communication Theory Workshop, and and General Chair of the Fifth International Symposium on Wireless Personal Multimedia Communications (WPMC'2002). He is a past Editor for Spread Spectrum with the IEEE Transactions on Communications (1993-1998), and a past member of the IEEE Communications Society Awards Committee (1999-2002). He is currently an elected member of the IEEE Vehicular Technology Society Board of Governors (2001-2003). 